\title{
Recommendations on hepatitis C screening for adults
}

\author{
Canadian Task Force on Preventive Health Care*
}

Cite as: CMAJ 2017 April 24;189:E594-604. doi: 10.1503/cmaj.161521

See related article at www.cmaj.ca/lookup/doi/10.1503/cmaj.170274

CMAJ Podcasts: author interview at https://soundcloud.com/cmajpodcasts/161521-guide

*The complete list of authors appears at the end of the article. The list of current members of the Canadian Task Force on Preventive Health Care is available at http://canadiantaskforce.ca/about/members.

A n estimated $0.64 \%-0.71 \%$ of Canadians (220000-245000 people) have chronic hepatitis $\mathrm{C}$ virus (HCV) infection, ${ }^{1}$ and approximately $44 \%{ }^{2}$ of those may be undiagnosed. $\mathrm{HCV}$ can be transmitted directly through percutaneous exposure (e.g., through inadequately sterilized medical equipment) or through receipt of contaminated blood products. ${ }^{3}$ People who inject drugs are at highest risk, but recipients of unscreened blood products, tissues or organs and patients undergoing long-term hemodialysis are also at increased risk. ${ }^{3}$ Less common modes of transmission include vertical transmission, high-risk sexual contact, unsterilized tattoo or piercing equipment, and occupational exposure. ${ }^{3}$ Not all people with chronic HCV infection will develop cirrhosis or signs or symptoms indicative of liver disease. ${ }^{4}$ It is estimated that approximately $84 \%$ of people infected with HCV do not develop cirrhosis 20 years after acute infection, and $59 \%$ after 30 years. ${ }^{5,6}$ Progression of liver fibrosis is variable and influenced by factors such as alcohol consumption, age at time of infection, male sex and HIV coinfection. ${ }^{\text {? }}$

Efficacy of treatment for HCV is typically evaluated using sustained virologic response (SVR), a surrogate outcome. ${ }^{8}$ Sustained virologic response is defined as having undetectable $\mathrm{HCV}$ ribonucleic acid (RNA) at 12, 24 or 72 weeks following completion of treatment (SVR12, SVR24 or SVR72). Historically, HCV treatment involved 24-48 weeks of pegylated interferon injections and oral ribavirin (pegylated interferon-ribavirin), which had substantial adverse effects. ${ }^{9}$ In 2011, Health Canada approved the use of direct-acting antiviral drugs (DAAs) in combination with pegylated interferon-ribavirin, which improved the likelihood of sustained virologic response but increased adverse effects. ${ }^{10,11}$ In 2016, Health Canada approved the use of pangenotypic interferon-free DAA regimens ${ }^{12}$ for treatment of HCV. Interferonfree DAA regimens are very costly, but have several advantages: they are administered orally, require shorter treatment duration and have higher likelihood of sustained virologic response, with fewer adverse effects than older regimens. ${ }^{9}$

\section{KEY POINTS}

- A systematic review found no evidence on the effectiveness of screening for HCV in the asymptomatic adult population.

- The task force recommends against screening for HCV in asymptomatic Canadian adults (including baby boomers) who are not at elevated risk of HCV infection.

- A strong recommendation against screening is warranted given its uncertain benefits but the certainty that it would lead to high levels of resource consumption. Referring individuals with screen-detected HCV for assessment would reduce access to assessment and treatment for people with clinically evident HCV.

- Other guidelines recommend testing individuals at elevated risk of $\mathrm{HCV}$, including immigrants from HCV-endemic countries, individuals with current or past history of injection drug use, incarcerated individuals, and those who received blood or blood products prior to 1992, among others.

There are no organized HCV screening programs for the general population in Canada, ${ }^{13}$ although the Public Health Agency of Canada (PHAC) and the College of Family Physicians of Canada (CFPC) recommend testing for hepatitis $\mathrm{C}$ in people at elevated risk for HCV. ${ }^{14}$

\section{Scope}

The Canadian Task Force on Preventive Health Care has not previously developed a recommendation on screening for HCV. Reasons for developing this recommendation include the availability of new treatments for chronic HCV infection and the lack of Canadian guidelines for screening. ${ }^{13}$ The current recommendations are intended to provide clinicians and policy-makers with guidance on screening asymptomatic Canadian adults for HCV. 


\section{Methods}

The task force is an independent panel of clinicians and methodologists that makes recommendations about clinical interventions aimed at primary and secondary prevention. These recommendations were developed by a workgroup of seven members of the task force with scientific support from PHAC. ${ }^{15}$ The recommendations were informed by two independently conducted systematic reviews ${ }^{16,17}$ that addressed specific aspects of the guideline's analytic framework (Appendix 1, available at www.cmaj.ca/ lookup/suppl/doi:10.1503/cmaj.161521/-/DC1). The reviews excluded post-transplant patients, patients with HIV, patients on hemodialysis and patients with occupational exposure; studies on all other population groups were sought, including higher-risk groups (e.g., with history of injection drug use) and higherprevalence groups (e.g., birth cohort).

The first set of reviews was conducted by the Canadian Agency for Drugs and Technologies in Health (CADTH) ${ }^{16}$ and attempted to identify evidence on 1 ) the effectiveness (benefits and harms) of HCV screening on HCV-related mortality and morbidity, including cirrhosis, hepatocellular carcinoma, rate of liver transplantation, quality of life, sustained virologic response rates, histological improvement, behavioural changes to improve health outcomes, reduced HCV transmission, anxiety, labelling, partner discord, abuse or violence, and overdiagnosis or overtreatment; 2 ) patient preferences and values in relation to screening; 3) the clinical validity of screening tests; and 4) the cost-effectiveness of screening. The peer-reviewed literature search was conducted in MEDLINE, Embase, PubMed and the Cochrane Library. For research questions on clinical effectiveness (benefits and harms), cost-effectiveness, and patient preferences and values, the search time frame was from January 2000 to March 2016; no date filters were applied to the question on the clinical validity of screening tests, which was run in June 2016. For all review questions, grey literature was sought using CADTH's Grey Matters checklist up to September 2016.

PHAC conducted a separate systematic review, ${ }^{17}$ which focused on the effectiveness of newer HCV treatments compared with older treatments, on mortality (all-cause or hepatic), cirrhosis (compensated or decompensated), hepatocellular carcinoma, need for liver transplantation, quality of life, sustained virologic response, improvement in liver histology, reduced HCV transmission, withdrawals due to adverse events, neutropenia, anemia, psychological adverse events, flu-like symptoms and rash. ${ }^{9,15,18}$ The literature search was an update to a therapeutic review conducted by CADTH in February 2015, which was further expanded by PHAC to include PubMed and ClinicalTrials.gov to November 2015.

Prepublication searches were conducted to update both reviews. The literature search for the screening review and its grey literature search were updated to Dec. 11, 2016. The literature update for the treatment review was updated to Nov. 21, 2016, but was limited to randomized controlled trials (RCTs) published in MEDLINE, Embase and the Cochrane Library.

The Knowledge Translation group of St. Michael's Hospital (Toronto, Ontario) engaged members of the public on behalf of the task force at two stages of the guideline development process. ${ }^{19,20}$ For phase one, 19 participants from the HCV screening and treatment populations rated outcomes to be included in the systematic reviews, using two online surveys and a focus group. ${ }^{19}$ For phase two, 15 asymptomatic, average-risk or increased-risk participants were recruited to provide their perception of the importance of considering harms, benefits and costs in making screening decisions. ${ }^{20}$

A modelling study ${ }^{21}$ was also commissioned by the task force and conducted by a team from the Toronto Health Economics and Technology Assessment Collaborative. This modelling study was used to examine the possible impact of screening under certain circumstances on hepatic mortality, hepatocellular carcinoma and decompensated cirrhosis (Appendix 2, available at www.cmaj.ca/ lookup/suppl/doi:10.1503/cmaj.161521/-/DC1). ${ }^{21}$ In the absence of empirical data, expert opinion was sought for four key model input parameters: 1) expected uptake of screening in the general population, 2) uptake of treatment in asymptomatic individuals, 3) genotype distribution, and 4) distribution of hepatic fibrosis scores in a primary care setting. Working group members identified five HCV experts, three of whom provided parameter estimates and ranges and the location of possible supplemental data supporting their estimates. Parameter estimates were established by calculating the mean value of the responses that the experts provided. The latest prevalence estimates from PHAC (2014) were used as inputs for the model for the general population and various subgroups., ${ }^{1,21}$

The Feasibility, Acceptability, Cost, and Health Equity (FACE) tool was used with organizational stakeholders to gain their perspective on the priority, feasibility, acceptability, cost and equity of the recommendation. ${ }^{22}$ The FACE survey was pilot-tested with this guideline as part of a validation exercise. Stakeholder organizations that provided input on the recommendation using the FACE survey are listed in Appendix 3 (available at www.cmaj.ca/ lookup/suppl/doi:10.1503/cmaj.161521/-/DC1).

The Grading of Recommendations Assessment, Development and Evaluation (GRADE) system was used to determine the quality of evidence and strength of recommendations ${ }^{18}$ (Box 1). The recommendations were revised and approved by the entire task force and underwent external review by academic and clinical experts. More information about task force methods can be found elsewhere. ${ }^{9,15,23}$ The task force plans to update this recommendation as new evidence or other compelling information about key factors influencing the recommendation becomes available (see Rationale section for details).

\section{Recommendations}

We recommend against screening for $\mathrm{HCV}$ in adults who are not at elevated risk (strong recommendation, very low-quality evidence).

The recommendation has been graded according to the GRADE system described in Box 1 . A summary of the recommendations for clinicians and policy-makers is shown in Box 2 .

\section{Screening}

The screening systematic review ${ }^{16}$ found no studies (RCTs, nonrandomized studies with a control group, or disease progression modelling studies) of the effectiveness of HCV screening in the 


\section{Box 1: Grading of recommendations}

Recommendations are graded according to the Grading of Recommendations Assessment, Development and Evaluation system (GRADE).$^{18}$ GRADE offers two strengths of recommendation: strong and weak. The strength of recommendations is based on the balance between desirable and undesirable outcomes; the confidence in the magnitude of the estimates of effect of the intervention on outcomes; the confidence in values and preferences and their variability; and whether the intervention represents a wise use of resources.

Strong recommendations are those for which the task force is confident that the desirable effects of an intervention outweigh its undesirable effects (strong recommendation for an intervention) or that the undesirable effects of an intervention outweigh its desirable effects (strong recommendation against an intervention). A strong recommendation implies that most individuals will be best served by the recommended course of action and that the recommendation can be adopted in practice or as policy in most situations.

Strong recommendations are normally based on high-quality evidence (i.e., high confidence in the estimate of the effect of an intervention). Strong recommendations may recommend in favour of an intervention (when there is high confidence of benefit) or against an intervention (when there is high confidence of harm). However, there are five circumstances in which the Task Force may consider a strong recommendation based on low- or very lowquality evidence: ${ }^{80}$

1) When low-quality evidence suggests benefit in a life-threatening situation (evidence regarding harms can be low or high)

2) When low-quality evidence suggests benefit and high-quality evidence suggests harm or a very high cost

3) When low-quality evidence suggests equivalence of two alternatives, but high-quality evidence of less harm for one of the competing alternatives

4) When high-quality evidence suggests equivalence of two alternatives and low-quality evidence suggests harm in one alternative

5) When high-quality evidence suggests modest benefits and low-/ very low-quality evidence suggests possibility of catastrophic harm

Weak recommendations are those for which the desirable effects probably outweigh the undesirable effects (weak recommendation for an intervention) or undesirable effects probably outweigh the desirable effects (weak recommendation against an intervention), but appreciable uncertainty exists. Weak recommendations result when the balance between desirable and undesirable effects is small, the quality of evidence is lower, or there is more variability in the values and preferences of patients. In cases where the balance of cost and benefits is ambiguous, key stakeholders differ about the acceptability or feasibility of implementation, and the effects on health equity are unclear are likely to result in a weak recommendation. A weak recommendation implies that most people would want the recommended course of action, but that many would not. For clinicians, this means that they must recognize that different choices will be appropriate for each individual, and that they must help each person arrive at a management decision consistent with his/her values and preferences. Policy-making will require substantial debate and involvement of various stakeholders.

Evidence is graded as high, moderate, low or very low quality, based on how likely further research is to change our confidence in the estimate of effect.
Box 2: Summary of recommendations for clinicians and policy-makers

We recommend against screening for HCV in adults who are not at elevated risk (strong recommendation, very low-quality evidence).

This recommendation applies only to adults who are not at elevated risk for HCV. It does not apply to pregnant women ${ }^{24}$ or adults who are at elevated risk for hepatitis $C,{ }^{14}$ such as:

- Individuals with current or history of injection drug use

- Individuals who have been incarcerated

- Individuals who were born, travelled or resided in HCV-endemic countries (Appendix 6)

- Individuals who have received health care where there is a lack of universal precautions

- Recipients of blood transfusions, blood products or organ transplant before 1992 in Canada

- Patients on hemodialysis

- Individuals who have had needle-stick injuries

- Individuals who have engaged in other risks sometimes associated with HCV exposure, such as high-risk sexual behaviours, homelessness, intranasal and inhalation drug use, tattooing, body piercing or sharing sharp instruments or personal hygiene materials with someone who is HCV positive

- Anyone with clinical clues suspicious for HCV infection (and above risk factors)

general population or in any other higher-risk or higherprevalence subgroup, including the 1950-1975 birth cohort. Only one uncontrolled retrospective study ${ }^{25}$ was found that reported on harms. That study ${ }^{25}$ was a retrospective review of records from a large urban US Department of Veterans Affairs hospital; the study reported that of 12485 people screened for HCV in 2001, only one patient experienced serious harm (hospitalization for pain control following liver biopsy).

The modelling study commissioned by the task force ${ }^{21}$ suggested that under the assumptions of the model, one-time screening of 100000 individuals not at elevated risk of HCV $(0.2 \%$ prevalence) could result in 199 new diagnoses of chronic HCV infection, compared with 91 persons identified through case finding (testing for HCV in individuals who show signs or symptoms or who are suspected of exposure), over a lifetime horizon (i.e., the model assumes that cases are ascertained across the lifespan of all 100000 simulated individuals). ${ }^{21}$ Assuming $89 \%$ of identified cases would receive treatment, screening was estimated to prevent 26 cases of decompensated cirrhosis and 20 cases of hepatocellular carcinoma, contributing to about 40 lives saved over a lifetime horizon per 100000 screened. ${ }^{21}$ Given the natural history of chronic HCV infection, this model showed that the expected benefit from screening would not be realized for 20-30 years from time of initial infection, with only $3 \mathrm{HCV}$-related deaths prevented at 5 years and 6 deaths at 10 years ${ }^{26}$ after screening 100000 individuals not at elevated risk.

The model ${ }^{21}$ has several important limitations that contribute to the uncertainty in the estimate of the effect: 1) an inability to consider potential differences in long-term outcomes between initiating treatment at earlier versus later stages of liver fibrosis, 
2) the possibility that the baseline risk of disease progression used for the model (based on nonscreened populations) ${ }^{27}$ is higher than that for the asymptomatic screened population, ${ }^{4,28}$ which would translate into an overestimation of the benefit of screening, 3) an inability to take into consideration the harms of overdiagnosis and overtreatment that result from screening, and 4) the inherent inability of modelling to account for unknown factors that may influence screening outcomes.

The screening review ${ }^{16}$ found low-quality evidence from 26 cross-sectional studies on the clinical validity of HCV screening tests (anti-HCV antibody and HCV-antigen tests) in predicting active infection (measured with a confirmatory HCV RNA test). ${ }^{29-54}$ Anti$\mathrm{HCV}$ antibody tests detect both active and past infection; therefore, individuals with past and resolved infections screen positive although they do not have active or chronic HCV infection. The proportion of confirmed active infections among positive screening test results varies widely across studies and test types. The proportion of RNA-positive, active HCV infection cases ranged from $0 \%$ to $89.7 \%$ among positive antibody-based assays ${ }^{29-43,45-48,51-54}$ and from $0 \%$ to $100 \%$ among antigen-based assays. ${ }^{39,44,49,50}$

Only three studies conducted confirmatory polymerase chain reaction on samples that tested negative for $\mathrm{HCV}$ antibody or antigen. The proportion of negative anti-HCV assays that were confirmed with polymerase chain reaction to be RNA negative ranged from $73.7 \%$ to $99.7 \%$ for two antibody assays ${ }^{29,39}$ and $89.7 \%$ in one antigen assay. ${ }^{32}$ The wide variation in results may be as a result of actual differences in test performance, varying study sample sizes or underlying variation in population characteristics, including population prevalence.

\section{Treatment}

Indirect evidence on treatment of HCV from the treatment review ${ }^{17}$ examined the benefits and harms of pegylated interferon-ribavirin treatment regimens compared with newer DAA-based regimens. This evidence ${ }^{17}$ is indirect, because the population examined is not a screened population and includes symptomatic individuals, who (by definition) are not the target of a screening program. ${ }^{17}$ Moderate-quality evidence from seven RCTs $(n=2431)^{55-61}$ showed that treatment with DAA-based regimens achieved higher sustained virologic response rates than PR at 48 weeks following treatment (SVR48) and reduced the frequency of harms (Appendix 4, available at www.cmaj.ca/lookup/ suppl/doi:10.1503/cmaj.161521/-/DC1). ${ }^{17}$ Very low-quality evidence showed no difference in quality of life (three RCTs, $n=$ $1342)^{62-64}$ or all-cause mortality ${ }^{55-57,61,65}$ at 36 to 72 weeks following treatment (five RCTs; $n=1853$ ) (Appendix 4). ${ }^{17}$ No studies reported on hepatic mortality, cirrhosis, hepatocellular carcinoma, need for liver transplant, and improvement in liver histology or reduced HCV transmission. Three RCTs $s^{55,57,65}$ suggested that there may be higher rates of SVR12 when treatment is given at an earlier fibrosis stage (F0 to F2) versus a later stage (F3 to F4). However, this was a post hoc analysis in which no statistical tests were done and that may serve as hypothesis generating only.

The evidence from the treatment review ${ }^{17}$ has several key limitations that reduce its applicability to this guideline. 1) Study participants were not identified by screening and could differ from people detected by population-based screening. It is uncertain whether the effects of treatment would be similar for symptomatic and screened, asymptomatic patients. ${ }^{17}$ 2) The identified studies did not include a comparison to an untreated control group. ${ }^{17} 3$ ) Evidence of benefit associated with newer treatments was restricted to surrogate outcomes assessed following a relatively short interval, given the chronic nature of HCV infection, ${ }^{17}$ and did not consider the potential risk of occurrence of hepatocellular carcinoma after achieving sustained virologic response. ${ }^{66}$

\section{Patient values and preferences}

The screening review ${ }^{16}$ found moderate- to low-quality evidence from 12 observational studies, ${ }^{67-74}$ which reported on patient preferences and values related to the decision to be screened for $\mathrm{HCV}$. Findings were highly variable in terms of patient preferences related to screening and there was a high level of uncertainty about the value that patients place on the clinical outcomes considered in the review. Concerns related to stigma and to access to care were reported as important factors for decision-making. ${ }^{67-74}$

A survey of 15 patients commissioned by the task force generally reinforced these findings and found that participants placed equal value on the benefits and harms of screening. ${ }^{20}$ Reduced mortality was perceived as a very important benefit, and concerns were noted about stigma and psychological adverse events from positive results of screening tests.

\section{Resource use}

A recent study examined the potential budget impact of $\mathrm{HCV}$ screening in Alberta. ${ }^{75}$ Assuming a $0.69 \%$ HCV prevalence, $70 \%$ screening uptake and $24 \%$ treatment uptake (reflects uptake common with older treatments, which led to more severe adverse events) and hypothetical negotiated drug costs of about $50 \%$ off list price, screening and treating the general population of Alberta was estimated to cost $\$ 253$ million over one year. This estimate was driven primarily by drug costs. ${ }^{75}$ If treatment uptake were to increase from $24 \%$ to $75 \%$ based on the use of DAA-based regimens with fewer adverse effects, the cost of screening and treatment would increase to $\$ 501$ million. ${ }^{75}$ Extrapolating these findings to the Canadian population not at elevated risk for HCV ( $n=19855629)$, and assuming an HCV prevalence of $0.2 \%$, a screening uptake of $70 \%$, treatment uptake of $75 \%$, and a hypothetical negotiated drug cost of about $50 \%$ off list price, would yield an estimated cost of more than $\$ 844$ million for screening only and approximately $\$ 1.5$ billion to screen and treat with DAA-based regimens. For the Canadian extrapolation, only $75 \%$ treatment uptake is used because it is more likely to reflect the situation going forward, when DAA-based regimens will be primarily used.

\section{Feasibility, acceptability, cost and equity}

The majority of individuals identified by screening are anticipated to be asymptomatic and in early stages of HCV (F0 to F1). At the time of writing this guideline, certain provinces had negotiated a price reduction with pharmaceutical companies that 
produce DAA-based treatment regimens. However, more advanced fibrosis (F2 to F4) and presentation with comorbidities is still needed to qualify for treatment in Canada. ${ }^{76,77}$ As a result, it is likely that most individuals who would be identified by screening would not qualify for drug coverage under their provincial plan. Given the high listed cost of DAA-based regimens (based on 2015 list prices in Alberta for a course of treatment: sofosbuvir + pegylated interferon-ribavirin, \$104429; ledipasvir + sofosbuvir $\$ 95000),{ }^{75}$ few people with HCV are likely to have the funds to pay out of pocket for a course of treatment. Therefore, a recommendation in favour of screening would increase the number of people with known HCV (and who are potentially susceptible to harms of stigma and anxiety) who could not access treatment, thus deriving no clear benefit despite the potential for harm from a diagnosis combined with treatment ineligibility. To the extent that wealthier individuals with screen-detected HCV could access treatment, a recommendation for screening has potential to increase health inequity. ${ }^{21}$

Presently, the lack of health system resources required to successfully roll out a treatment strategy that includes all individuals with HCV means that population-based screening is unlikely to be acceptable to funders such as provincial and territorial governments, especially given the uncertain benefits of a screening program. The results of the FACE survey are presented in Appendix 3.

Data from 38 countries show that the price of DAA medication varies substantially between lower- and higher-income countries and even across high-income countries. For example, one bottle of sofosbuvir is reported to cost (in USD) \$161 to \$312 in India, $\$ 500$ in Ivory Coast, $\$ 14000$ in Spain and \$20590 in Switzerland. ${ }^{78}$ These set costs are not correlated with gross national income or with manufacturing costs. The task force encourages policymakers to continue to work with pharmaceutical companies to offer affordable prices for new HCV treatments, which might justify the allocation of the resources required for a successful rollout of a treatment strategy for all individuals identified with HCV, including those with no comorbidities in the early stages of liver disease (F0 and F1). If pharmaceutical companies were to decrease substantially the cost of new HCV drugs for all people diagnosed with early stages of fibrosis (i.e., F0 and F1) regardless of comorbidity, and policy-makers were to confirm that health system resources are in place to ensure a successful rollout of a treatment strategy, it is possible that under such circumstances, the resources spent on screening individuals not at elevated risk for HCV could be worth the expected net benefit, even if that benefit were small.

\section{Rationale}

The screening review ${ }^{15}$ did not identify any evidence for the benefit of screening for HCV on critical outcomes. The modelling study estimated possible results for three critical outcomes (hepatic mortality, hepatocellular carcinoma and decompensated cirrhosis), but this model was rated as very low-quality evidence. Given the lack of direct evidence on screening for HCV and the many assumptions required by the model, the task force considers the overall quality of evidence supporting this recommendation to be very low (i.e., highly uncertain). Given the prevalence of HCV in
Canadians not at elevated risk for HCV, the lack of direct evidence on the benefits and harms of screening in all groups of the population, and the very low quality of the indirect evidence produced by modelling, substantial uncertainty remains about the effectiveness of screening among adults in Canada.

This recommendation places a relatively lower value on 1) very low-quality indirect evidence suggesting a potentially small benefit from screening, 2) the low risk of household and sexual transmission of HCV among individuals not at elevated risk, as well as the low risk of transmission through blood products given routine screening of blood and organs, ${ }^{79}$ and 3 ) the potential risk of the individual developing end-stage liver disease and transmitting the infection despite being asymptomatic.

On the other hand, this recommendation places a relatively higher value on 1) the anticipated increase in harm resulting from diagnosing and treating individuals who screen positive but would have never developed HCV-related disease during their lifetime; 2) false positives and false negatives, which could lead to unnecessary anxiety and/or false reassurance; 3) the potential for screening to increase inequity, given that among those who do not meet current eligibility criteria (e.g., specific comorbidities), only wealthier individuals or those with private insurance would obtain earlier access to treatment not currently funded by government; 4) the unknown magnitude of benefit of treatment on reducing risk of transmission; and 5) the very large impact that screening and treatment would have on health care budgets, and associated opportunity costs (i.e., the limit this would place on the ability to provide other health care interventions that would have to be forgone for lack of funds, despite being supported by better evidence).

Indirect evidence showing that new DAA-based treatment is much more effective in achieving sustained virologic response than older pegylated interferon-ribavirin treatment regimens was also considered in developing this recommendation. As such, emerging evidence on new treatments with similar effectiveness would not change this recommendation. Our recommendation is also informed by limited evidence on patient preferences: although some patients would want to be screened, many would forgo treatment if it were not funded by provincial and territorial drug plans. The rationale for this recommendation is outlined in the GRADE Evidence to Decision framework (Appendix 5, available at www.cmaj.ca/lookup/suppl/doi:10.1503/ cmaj.161521/-/DC1).

The recommendation is strong because the task force is confident of the potential for harm resulting from screening and treatment for HCV, and substantial uncertainty remains about the benefits of screening. Considering the totality of evidence, this means that the estimate of beneficial effect could be substantially different from the true effect of screening. The task force is confident that implementing any recommendation to screen and treat those identified as HCV positive would require substantial health system resources. This burden would limit the ability of clinicians to deliver other interventions of proven value. ${ }^{80}$

Further, because of the degree of uncertainty related to any benefit of screening, it would be difficult to define a set of circumstances under which a practitioner should offer screening to an 
individual who is not at increased risk for HCV. The strong recommendation implies that the majority of such patients will be better served by not being screened for HCV. Considering that funding for treatment is presently limited to individuals with identified HCV and more advanced liver fibrosis (F2 to F4), the task force believes our recommendation should allow clinicians to focus on providing treatment to individuals who have already been diagnosed.

This guideline may be re-evaluated as evidence on the benefit and/or harm of screening emerges, such as evidence examining the real-life consequences of screening and treating those who would have otherwise never developed complications or died from liver disease; or as other factors influencing the recommendations change, such as improved access to treatment. This could occur, for instance, if there were a substantial reduction in the price of treatment, to an extent that would permit all individuals with HCV to be treated. The task force notes that given the potential impact of treatment on budget, even very large reductions in price might not be sufficient to recommend screening. Even if markedly lower drug prices were available, changes to models of care may also be required before population-based screening could be warranted, such as changes in health system policies to support a successful rollout of a treatment strategy that would include all individuals identified as having chronic $\mathrm{HCV}$ infection, regardless of fibrosis stage or comorbidity. Better access to DAA-based treatment may require extending management of HCV to clinicians in primary care.

\section{Considerations for implementation}

The task force recommendation applies to individuals who are not pregnant or at elevated risk for HCV. Subgroups of the population who are at increased risk for HCV (and not included in this recommendation) may require special attention from clinicians. A joint 2009 recommendation from CFPC and PHAC, ${ }^{14}$ although not based on a systematic review of the evidence, addressed those individuals who are at increased risk. That guidance suggests testing for HCV in "anyone with risk behaviours for HCV, with potential exposure to $\mathrm{HCV}$, and/or with clinical clues suspicious for HCV." Populations targeted in the CFPC/PHAC guideline ${ }^{14}$ include people who inject drugs (currently or in the past); individuals who have been incarcerated; individuals who may have been exposed to contaminated blood, blood products or medical equipment; and those who have travelled or resided in endemic regions. ${ }^{14,81,82}$

Some immigrants are at increased risk for HCV because they are from countries where HCV infection is common. Unlike the nonimmigrant population, these persons are at increased risk for $\mathrm{HCV}$ because of iatrogenic exposure in their country of origin (e.g., lack of standard precautions, or as a result of medical or dental procedures with contaminated equipment) and not necessarily from injection drug use or other higher-risk behaviours. ${ }^{83}$ The CFPC/PHAC guidance ${ }^{14}$ recommends testing for HCV in individuals who were "born, traveled or resided in a region in which $\mathrm{HCV}$ infection is more common." A list of endemic countries and a related map are provided in Appendix 6 (available at www. cmaj.ca/lookup/suppl/doi:10.1503/cmaj.161521/-/DC1).
More persons in subgroups such as the Indigenous population (3\% prevalence) ${ }^{1}$ and the cohort born from 1950 to 1975 (0.8\% prevalence) are diagnosed with chronic $\mathrm{HCV}^{2}$ these populations have a higher proportion of individuals at higher risk for $\mathrm{HCV}$ because of identifiable risk factors. ${ }^{1,2,7,84,85}$ For example, removing from the Indigenous population people who inject drugs would reduce the $\mathrm{HCV}$ prevalence from $3 \%$ to $0.5 \% .{ }^{1,85}$ Individuals from the Indigenous population who are not otherwise at increased risk are, therefore, included in the present task force guidance, which recommends against screening adults who are not at elevated risk. Similarly, the increased reported prevalence in the cohort born between 1950 and 1975 is likely driven by an increased prevalence of risk behaviours or potential exposures, rather than birth year per se. ${ }^{7,84}$ In the judgment of the task force, neither Indigenous people nor members of the 1950-1975 birth cohort should be screened for HCV in the absence of other characteristics that would place them at increased risk for HCV.

The task force considered the possibility of screening a birth cohort; that is, one-time testing of all people born, for example, between 1950 and 1975. Most individuals in the birth cohort who are at elevated risk are included in the joint CFPC/PHAC guideline. ${ }^{14}$ Following this risk-based guideline will likely increase the identification of those who will benefit most from testing. Those born from 1950 to 1975, who are not otherwise at increased risk, are included in the present task force guidance, which recommends against screening adults who are not at elevated risk. More evidence would be needed before making a recommendation about birth cohort testing, separate from adults in the general population.

The task force developed knowledge translation tools to help clinicians assess their patients' risk for HCV, so that testing can be offered to those at increased risk. These are available at http://canadiantaskforce.ca/tools-resources/hepatitis-c-2/.

\section{Monitoring and evaluation}

Given that the task force has recommended against screening adults who are not at elevated risk of HCV, a clear indicator of the uptake of this guideline would be decreased screening of individuals who do not present with risk factors.

\section{Other guidelines}

Population-based screening for HCV is not recommended by the task force. The task force recommendation aligns with recently published clinical guidelines from the World Health Organization; ${ }^{86,87}$ National Institute for Health and Care Excellence; ${ }^{88}$ Scottish Intercollegiate Guidelines Network; ${ }^{89}$ Immigration, Refugees and Citizenship Canada; ${ }^{90}$ UK National Screening Committee; ${ }^{91}$ and the Gastroenterological Society of Australia. ${ }^{92}$ It partly aligns with guidelines from the Canadian Collaboration for Immigrant and Refugee Health, ${ }^{93}$ the US Preventive Services Task Force (USPSTF) ${ }^{94}$ and the US Centers for Disease Control and Prevention (CDC) $(1998)^{95}$ (Table 1). Although there is variation in definitions, most jurisdictions recommend some sort of risk-based testing.

The more recent guideline from CDC (2012) ${ }^{96}$ and the USPSTF ${ }^{94}$ guideline recommend one-time screening for those born between 
1945 and 1965. This recommendation relies on indirect evidence such as prevalence (estimated to be $3.25 \%$ in the US, ${ }^{96}$ which is four times higher than in Canada at $0.8 \%{ }^{2}$ ), attainment of sustained virologic response (a surrogate outcome) and the ability of practitioners to influence screening uptake. The CDC and USPSTF recommendations acknowledge the lack of direct evidence on effectiveness of screening in this cohort and the potential for screening to increase overall harms in this population ${ }^{97,98}$ related to overdiagnosis and overtreatment.

\section{Gaps in knowledge}

An important gap is the lack of studies that examine the benefits, harms and other potential consequences of screening asymptomatic populations. RCTs that compare treatment at earlier versus later stages of liver fibrosis are needed. A large population-based prevalence study of chronic HCV in Canada is also lacking. Small studies, primarily in lower- or higher-risk groups and using modelled data, are available, but the confidence in the certainty of those

Table 1 (part 1 of 2): National and international guidelines on testing and screening for hepatitis C virus*

\section{Organization}

Canadian Task Force on Preventive Health Care (current guideline, 2017)

US Centers for Disease Control and Prevention (1998) ${ }^{95}$

College of Family Physicians of Canada and the Public Health Agency of Canada $(2009)^{14}$

UK National Screening Committee $(2011)^{91}$

Canadian Collaboration for Immigrant and Refugee Health (2011) ${ }^{93}$

US Centers for Disease Control and Prevention (2012) ${ }^{96}$

Immigration, Refugees and Citizenship Canada (2013) ${ }^{90}$

\section{Recommendation}

We recommend against screening for HCV in adults who are not at elevated risk (strong recommendation, very low-quality evidence). This recommendation applies only to adults who are not at elevated risk for HCV. It does not apply to pregnant women or adults who are at elevated risk for hepatitis $\mathrm{C}$, such as individuals with current or history of injection drug use; individuals who have been incarcerated; individuals who were born, travelled or resided in HCV-endemic countries; individuals who have received health care where there is a lack of universal precautions; recipients of blood transfusions, blood products or organ transplant before 1992 in Canada; patients on hemodialysis; individuals who have had needle-stick injuries; individuals who have engaged in other risks sometimes associated with HCV exposure, such as high-risk sexual behaviours, homelessness, intranasal and inhalation drug use, tattooing, body piercing or sharing sharp instruments or personal hygiene materials with someone who is HCV positive; and anyone with clinical clues suspicious for HCV infection.

The Centers for Disease Control and Prevention recommends routine testing of those at high risk, defined as history of injection drug use, those at risk of health care-associated transmission, individuals with HIV, those with a recognized exposure, or those who are concerned. This is an unrated risk-based recommendation, based on expert opinion related to prevalence in risk groups

The College of Family Physicians of Canada and Public Health Agency of Canada guideline recommends screening anyone with risk behaviours or potential exposures, defined as injection drug use, incarceration, exposure in a high-prevalence region (born, travelled or resided), health care-associated transmission, higher-risk sexual behaviour, tattoos or body piercing, or ceremonial rituals requiring skin piercing done with suspect infection control, etc. Individuals with any of the above risk factors and the following should also be tested: abnormal ALT, HIV, hepatitis B, non-Hodgkin lymphoma, signs of chronic liver, etc. These are unrated risk-based recommendations, based on expert opinion.

The National Screening Committee recommended against a national screening program for HCV among people of ethnic minorities who are born outside the UK. This is an unrated recommendation based on a lack of randomized controlled trial data on the effectiveness of screening programs to reduce morbidly and mortality in addition to other areas related to program initiation.

The Canadian Collaboration for Immigrant and Refugee Health recommends screening all immigrants and refugees from regions with prevalence of disease $\geq 3 \%$ (this excludes South Asia, Western Europe, North America, Central America and South America). This prevalence-based recommendation is based on moderate-quality evidence factoring in increased risk of death from viral hepatitis and hepatocellular carcinoma in immigrants and refugees compared with the general population.

The Centers for Disease Control and Prevention augmented its 1998 guideline by recommending one-time testing without prior ascertainment of HCV risk for adults born between 1945 and 1965. This is a strong cohort-based recommendation based on moderate-quality evidence that these benefits outweigh harms: attainment of SVR, and SVR's association with reduced risk of hepatocellular carcinoma and all-cause mortality. Anecdotal evidence was also considered: undergoing liver biopsy, false-positive tests, anxiety, treatment access and effect of HCV status on insurance and employment. Cohort chosen was based on prevalence estimates.

Immigration, Refugees and Citizenship Canada recommends one-time screening of those with risk factors such as injection drug use; health care-associated transmission, including occupational exposure; higher-risk sexual behaviour; tattoos or body piercing; former incarceration; children born to mothers with chronic hepatitis $C$ infection; those with signs or symptoms of liver disease, active tuberculosis, HIV or syphilis, etc., with particular emphasis on those from endemic countries: Egypt, Pakistan and China are specifically mentioned. These are unrated risk-based recommendations, based on expert opinion and provincial recommendations (British Columbia) and the World Health Organization. 


\section{Organization}

National Institute for Health and Care Excellence $(2013)^{88}$

US Preventive Services Task Force $(2013)^{94}$

Scottish Intercollegiate Guidelines Network (2013) $)^{89}$

World Health Organization (2014) ${ }^{87}$

Gastroenterological Society of Australia $(2016)^{92}$

World Health Organization (2016) ${ }^{86}$
Recommendation

The National Institute for Health and Care Excellence recommends one-time testing of those at high risk including injection drug users, people at risk of health care-associated transmission, migrants from countries with a $2 \%$ or greater prevalence, incarcerated persons, the homeless, children or youth in care homes, HIV-positive men who have sex with men, etc. This is an unrated risk-based recommendation, based on moderate evidence that safe and responsible injecting practices are employed by injection drug users to avoid the transmission of hepatitis $\mathrm{C}$, and based on inferencederived evidence (expert opinion) on studies of hepatitis B.

The US Preventive Services Task Force recommends one-time screening of adults born between 1945 and 1965 and those at high risk of infection as a result of potential exposure before universal blood screening. It also recommends periodic screening of individuals with a history of risk exposure, such as injection drug users. This is a grade $B$ cohort- and risk-based recommendation, based on adequate evidence that harms are small, moderate benefit of screening test accuracy studies and moderate certainty of SVR as an intermediate outcome and its link to reduced hepatocellular carcinoma and mortality. The review did not find evidence on effect of current treatments and long-term outcomes, or evidence on benefit of screening asymptomatic adults in reducing morbidity or mortality. Cohort chosen was based on prevalence estimates.

The Scottish Intercollegiate Guidelines Network recommends one-time testing of individuals who might benefit from knowing their status and those with an excess risk, defined as injection drug users, people at risk of health care-associated transmission, those with HIV, people with tattoos or body piercing done with suspect infection control, elevated ALT, migrants from medium- or high-prevalence countries, etc. Individuals who remain at high risk should be offered annual testing. This is an expert opinion grade D risk-based recommendation, based on prevalence, other guidelines and a few studies on injection drug use.

In low- and middle-income countries, the World Health Organization recommends one-time screening of individuals who are at high risk of HCV, defined broadly to include people at risk of health careassociated transmission, individuals with HIV, injection drug users, etc. This is a strong risk-based recommendation, based on moderate-quality evidence of the influence of media and practitioners to increase uptake of screening. Studies did not consider harms and found no direct evidence showing that targeted testing increased SVR or reduced mortality.

The Gastroenterological Society of Australia recommends annual screening of HCV-seronegative people with risk factors for HCV transmission, defined as injection drug users, sex workers, people with tattoos or body piercing, people in custodial settings, people with HIV or hepatitis B, people who have evidence of liver disease, migrants from high-prevalence regions, etc. This is an expert opinion grade A1 risk-based recommendation based on prevalence and modelled reduction in liver-related deaths as a result of achieving SVR.

This recommendation is identical to 2014 recommendations. In low- and middle-income countries, the World Health Organization recommends one-time screening of individuals who are at high risk of HCV, defined broadly to include people at risk of health care-associated transmission, individuals with HIV, injection drug users, etc. This is a strong risk-based recommendation, based on moderate-quality evidence on the influence of media and practitioners to increase uptake of screening. Studies did not consider harms and found no direct evidence showing that targeted testing increased SVR or reduced mortality.

Note: $\mathrm{ALT}=$ alanine aminotransferase, $\mathrm{HCV}=$ hepatitis $\mathrm{C}$ virus, $\mathrm{HIV}=$ human immunodeficiency virus, $\mathrm{SVR}=$ sustained virologic response

${ }^{\star}$ This table reports only on HCV testing or screening component of guidelines.

estimates is low. Although there is some evidence on the natural history of HCV infection, ${ }^{5}$ there is uncertainty about the factors that influence the progression of liver disease and how these factors may affect the proportion of people who go on to develop end-stage liver disease, in some cases, despite achieving sustained virologic response. Although there are observational data examining the association of sustained virologic response with long-term outcomes important to patients, ${ }^{99,100}$ additional studies are needed to ascertain whether sustained virologic response associated with newer agents (DAAs) yields similar outcomes.

\section{Conclusion}

The HCV prevalence in most adults in the general Canadian population is low ${ }^{1}$ and direct evidence examining the benefits and harms of screening for HCV is not available. Thus, the task force recommends against screening adults who are not at elevated risk for HCV. Not screening for HCV will focus our limited health care resources to test (and treat) individuals at elevated risk for $\mathrm{HCV}$ and to provide other medical interventions that are proven to be of benefit. 


\section{References}

1. Trubnikov M, Yan P, Archibald C. Estimated prevalence of hepatitis C virus infection in Canada, 2011. Can Commun Dis Rep. 2014;40. Available: www.phac-aspc. gc.ca/publicat/ccdr-rmtc/14vol40/dr-rm40-19/surveillance-b-eng.php (accessed 2017 Apr. 6).

2. Rotermann M, Langlois K, Andonov A, et al. Seroprevalence of hepatitis B and C virus infections: results from the 2007 to 2009 and 2009 to 2011 Canadian Health Measures Survey. Cat no 82-003-X, Health Rep Vol 24, No 11;3-13. Ottawa: Statistics Canada; 2013. Available: www.statcan.gc.ca/pub/82-003-x/2013011/ article/11876-eng.pdf (accessed 2016 Nov. 1).

3. Healthy Canadians: risks of hepatitis C. Ottawa: Government of Canada; 2016. Available: https://www.canada.ca/en/public-health/services/diseases/ hepatitis-c/risks-hepatitis-c.html (accessed 2016 Nov. 1).

4. Seeff LB. The history of the "natural history" of hepatitis C (1968-2009). Liver Int 2009;29(Suppl 1):89-99.

5. Thein HH, Yi Q, Dore GJ, et al. Estimation of stage-specific fibrosis progression rates in chronic hepatitis $\mathrm{C}$ virus infection: a meta-analysis and meta-regression. Hepatology 2008;48:418-31.

6. Westbrook RH, Dusheiko G. Natural history of hepatitis C. J Hepatol 2014; 61(Suppl):S58-68.

7. Chen SL, Morgan TR. The natural history of hepatitis $\mathrm{C}$ virus (HCV) infection. Int J Med Sci 2006;3:47-52.

8. Innes HA, McDonald SA, Dillon JF, et al. Toward a more complete understanding of the association between a hepatitis $C$ sustained viral response and cause-specific outcomes. Hepatology 2015;62:355-64.

9. Recommendations: diagnosis of acute and chronic hepatitis C. Geneva: European Association for the Study of the Liver (EASL); 2015. Available: www.easl.eu/ research/our-contributions/clinical-practice-guidelines/detail/recommendations-on -treatment-of-hepatitis-c-2015/report/4 (accessed 2016 Oct. 18).

10. Wells G, Kelly S, Farah B, et al. Drugs for chronic hepatitis C infection: clinical review. CADTH Therapeutic Review, Vol 3. Ottawa: Canadian Agency for Drugs and Technologies in Health; 2016. Available: https://www.cadth.ca/sites/default/files/pdf/ TR0008_Clinical_Report-en.pdf (accessed 2016 Oct. 18).

11. Summary basis of decision for INCIVEK. Ottawa: Health Canada; 2012. Available: https://hpr-rps.hres.ca/reg-content/summary-basis-decision-detailOne. php?lang=en\&linkID=SBD00070 (accessed 2017 Apr. 6).

12. Regulatory decision summary for EPCLUSA. Ottawa: Health Canada; 2016. Available: https://hpr-rps.hres.ca/reg-content/regulatory-decision-summary -detail.php?lang=en\&linkID=RDS00181 (accessed 2017 Apr. 6).

13. Shah HA, Heathcote J, Feld JJ. A Canadian screening program for hepatitis C: is now the time? CMAJ 2013;185:1325-8.

14. Pinette G, Cox J, Heathcote J, et al. Primary care management of chronic hepatitis C: professional desk reference 2009. The College of Family Physicians of Canada (CFPC) and the Public Health Agency of Canada (PHAC); 2009. Available: www.cfpc.ca/uploadedFiles/Resources/Resource_Items/HEP_C_Guide_ eng_2.pdf (accessed 2016 Nov. 3).

15. Canadian Task Force on Preventive Health Care. Canadian Task Force on Preventive Care procedure manual. Calgary; 2014. Available: http://canadiantaskforce .ca/methods/ (accessed 2017 Apr. 6)

16. Screening for hepatitis C: a systematic review and meta-analysis. Ottawa: Canadian Agency for Drugs and Technologies in Health; 2016. Available: https://www. cadth.ca/screening-hepatitis-c-systematic-review-and-meta-analysis (accessed 2017 Feb. 27).

17. Reyes Domingo F, Holmes NM, Jaramillo Garcia A, et al. Treatment for hepatitis C virus: a systematic review and meta-analysis. Updated 7 April 2017. 2017. Available: http://canadiantaskforce.ca/guidelines/published-guidelines/hepatitis-c/.

18. Schünemann H, Brożek J, Guyatt G, et al., editors. GRADE handbook. The GRADE Working Group; 2013. Available: http://gdt.guidelinedevelopment.org/ app/handbook/handbook.html (accessed 2017 Apr. 6).

19. St. Michael's Hospital, Knowledge Translation Program, Li Ka Shing Knowledge Institute. CTFPHC patient preferences protocol, updated 26 Jan. 2015. http://canadiantaskforce.ca/methods (accessed 2017 Apr. 5).

20. Buckland D, Sayal R, Bashir N, et al. Patient preferences in considering hepatitis C screening and treatment outcomes: phase two. Toronto: Li Ka Shing Knowledge Institute, St. Michael's Hospital; 2016. Available: http://canadiantaskforce. ca/guidelines/published-guidelines/hepatitis-c/.
21. Wong W, Erman A, Krahn M. Model-based Projection of Health and Economic Effect of Screening Hepatitis C in Canada 2016 update - Final Report. November 22, 2016. Available: http://canadiantaskforce.ca/guidelines/publishedguidelines/hepatitis-c/.

22. Pottie K, Siu W, Duclos P, and the members of the World Health Organization Technical Consultation on Pain Migration (2016). New recommendations to prevent pain during immunizations. WHO Position Paper. 2015. Available: doi:10.1016/j.vaccine.2015.11.064.

23. Connor Gorber S, Singh H, Pottie K, et al. Process for guideline development by the reconstituted Canadian Task Force on Preventive Health Care. CMAJ 2012;184:1575-81.

24. SOGC Clinical Practice Guidelines. The reproductive care of women living with hepatitis C infection. Ottawa: The Society of Obstetricians and Gynaecologists of Canada; 2000. Available: https://sogc.org/wp-content/uploads/2013/01/ gui96ECPG0010wDisclaimer.pdf (accessed 2016 Dec. 15).

25. Groom H, Dieperink E, Nelson DB, et al. Outcomes of a hepatitis C screening program at a large urban VA medical center. J Clin Gastroenterol 2008;42:97-106.

26. Seeff LB. Natural history of chronic hepatitis C. Hepatology 2002;36(Suppl 1):S35-46.

27. Wong WW, Tu HA, Feld JJ, et al. Cost-effectiveness of screening for hepatitis C in Canada. CMAJ 2015;187:E110-21.

28. Koretz RL, Lin KW, loannidis JPA, et al. Is widespread screening for hepatitis C justified? BMJ 2015;350:g7809.

29. Lyons MS, Kunnathur VA, Rouster SD, et al. Prevalence of diagnosed and undiagnosed hepatitis $C$ in a midwestern urban emergency department. Clin Infect Dis 2016;62:1066-71.

30. Blaxhult A, Samuelson A, Ask R, et al. Limited spread of hepatitis C among HIVnegative men who have sex with men in Stockholm, Sweden. Int J STD AIDS 2014;25:493-5.

31. Sommese L, Sabia C, Paolillo R, et al. Screening tests for hepatitis B virus, hepatitis $C$ virus, and human immunodeficiency virus in blood donors: evaluation of two chemiluminescent immunoassay systems. Scand J Infect Dis 2014; 46:660-4.

32. Sommese L, lannone C, Cacciatore F, et al. Comparison between screening and confirmatory serological assays in blood donors in a region of South Italy. J Clin Lab Anal 2014;28:198-203.

33. Valois RC, Maradei-Pereira LM, Crescente JA, et al. HCV infection through perforating and cutting material among candidates for blood donation in Belém, Brazilian Amazon. Rev Inst Med Trop Sao Paulo 2014;56:511-5.

34. Baha W, Foullous A, Dersi N, et al. Prevalence and risk factors of hepatitis B and $C$ virus infections among the general population and blood donors in Morocco. BMC Public Health 2013;13:50.

35. Zeba MT, Sanou M, Bisseye C, et al. Characterisation of hepatitis C virus genotype among blood donors at the regional blood transfusion centre of Ouagadougou, Burkina Faso. Blood Transfus 2014;12(Suppl 1):s54-7.

36. Li D, Long Y, Wang $\mathrm{T}$, et al. Epidemiology of hepatitis $\mathrm{C}$ virus infection in highly endemic HBV areas in China. PLoS One 2013;8:e54815.

37. Martins T, Machado DF, Schuelter-Trevisol F, et al. Prevalence and factors associated with HCV infection among elderly individuals in a southern Brazilian city. Rev Soc Bras Med Trop 2013;46:281-7.

38. Woo GA, Hill MA, de Medina MD, et al. Screening for hepatitis B virus and hepatitis $C$ virus at a community fair: a single-center experience. Gastroenterol Hepatol (N Y) 2013;9:293-9.

39. Kesli R, Polat H, Terzi Y, et al. Comparison of a newly developed automated and quantitative hepatitis $\mathrm{C}$ virus (HCV) core antigen test with the HCV RNA assay for clinical usefulness in confirming anti-HCV results. J Clin Microbiol 2011;49:4089-93.

40. Kesli R, Ozdemir M, Kurtoglu MG, et al. Evaluation and comparison of three different anti-hepatitis $C$ virus antibody tests based on chemiluminescence and enzyme-linked immunosorbent assay methods used in the diagnosis of hepatitis C infections in Turkey. J Int Med Res 2009;37:1420-9.

41. Rashdan A, Hijjawi S, Jadallah K, et al. Prevalence of hepatitis C virus antibodies among blood donors in Northern Jordan. Jordan Med J 2008;42:179-83.

42. Reis NRS, Motta-Castro ARC, Silva ÁMC, et al. Prevalence of hepatitis C virus infection in quilombo remnant communities in Central Brazil. Rev Inst Med Trop Sao Paulo 2008;50:359-60. 
43. Slavenburg S, Verduyn-Lunel FM, Hermsen JT, et al. Prevalence of hepatitis C in the general population in the Netherlands. Neth J Med 2008;66:13-7.

44. Letowska M, Brojer E, Mikulska M, et al. Hepatitis C core antigen in Polish blood donors. Transfusion 2004;44:1067-71.

45. Dalgard O, Jeansson S, Skaug K, et al. Hepatitis C in the general adult population of Oslo: prevalence and clinical spectrum. Scand J Gastroenterol 2003; 38:864-70.

46. Zervou EK, Boumba DS, Liaskos C, et al. Low prevalence of HCV, HIV, and HTLV-I/II infection markers in northwestern Greece: results of a 3-year prospective donor study (1995-1997). Eur J Intern Med 2003;14:39-44.

47. Alberti A, Noventa F, Benvegnù L, et al. Prevalence of liver disease in a population of asymptomatic persons with hepatitis C virus infection. Ann Intern Med 2002;137:961-4.

48. Kondili LA, Chionne $\mathrm{P}$, Costantino A, et al. Infection rate and spontaneous seroreversion of anti-hepatitis $C$ virus during the natural course of hepatitis $C$ virus infection in the general population. Gut 2002;50:693-6.

49. Muerhoff AS, Jiang L, Shah DO, et al. Detection of HCV core antigen in human serum and plasma with an automated chemiluminescent immunoassay. Transfusion 2002;42:349-56.

50. Icardi G, Ansaldi F, Bruzzone BM, et al. Novel approach to reduce the hepatitis $C$ virus (HCV) window period: Clinical evaluation of a new enzyme-linked immunosorbent assay for HCV core antigen. J Clin Microbiol 2001;39:3110-4.

51. Maio G, d'Argenio P, Stroffolini T, et al. Hepatitis $C$ virus infection and alanine transaminase levels in the general population: a survey in a southern italian town. J Hepatol 2000;33:116-20.

52. Lucas RE, Faoagali JL. The serological status of Solomon Island blood donors. Southeast Asian J Trop Med Public Health 1999;30:542-5.

53. Guadagnino V, Stroffolini T, Rapicetta M, et al. Prevalence, risk factors, and genotype distribution of hepatitis $C$ virus infection in the general population: a community-based survey in southern Italy. Hepatology 1997;26:1006-11.

54. Vrielink H, Reesink HW, van den Burg PJ, et al. Performance of three generations of anti-hepatitis $C$ virus enzyme-linked immunosorbent assays in donors and patients. Transfusion 1997;37:845-9.

55. Fried MW, Buti M, Dore GJ, et al. Once-daily simeprevir (TMC435) with pegylated interferon and ribavirin in treatment-naïve genotype 1 hepatitis C: the randomized PILLAR study. Hepatology 2013;58:1918-29.

56. Hayashi N, Izumi N, Kumada H, et al. Simeprevir with peginterferon/ribavirin for treatment-naïve hepatitis C genotype 1 patients in Japan: CONCERTO-1, a phase III trial. J Hepatol 2014;61:219-27.

57. Jacobson IM, Dore GJ, Foster GR, et al. Simeprevir with pegylated interferon alfa 2a plus ribavirin in treatment-naive patients with chronic hepatitis $C$ virus genotype 1 infection (QUEST-1): a phase 3, randomised, double-blind, placebo-controlled trial. Lancet 2014;384:403-13.

58. Manns M, Marcellin P, Poordad F, et al. Simeprevir with pegylated interferon alfa $2 \mathrm{a}$ or $2 \mathrm{~b}$ plus ribavirin in treatment-naive patients with chronic hepatitis $C$ virus genotype 1 infection (QUEST-2): a randomised, double-blind, placebocontrolled phase 3 trial. Lancet 2014;384:414-26.

59. An efficacy, pharmacokinetics, safety and tolerability study of TMC 435 as part of a treatment regimen for hepatitis C-infected patients. no. NCT01725529. 2015. Available: https://clinicaltrials.gov/ct2/show/NCT01725529 (accessed 2016 Nov. 10).

60. Lawitz E, Lalezari JP, Hassanein T, et al. Sofosbuvir in combination with peginterferon alfa-2a and ribavirin for non-cirrhotic, treatment-naive patients with genotypes 1, 2, and 3 hepatitis $C$ infection: a randomised, double-blind, phase 2 trial. Lancet Infect Dis 2013;13:401-8.

61. Lawitz E, Mangia A, Wyles D, et al. Sofosbuvir for previously untreated chronic hepatitis C infection. N Engl J Med 2013;368:1878-87.

62. Wei L, Han T, Yang D, et al. Simeprevir plus peginterferon/ribavirin for HCV genotype 1-infected treatment-naïve patients in China and South Korea. J Gastroenterol Hepatol 2016;31:912-20.

63. Younossi ZM, Stepanova M, Henry L, et al. Minimal impact of sofosbuvir and ribavirin on health related quality of life in chronic hepatitis $\mathrm{C}(\mathrm{CH}-\mathrm{C})$. J Hepatol 2014;60:741-7.

64. Scott J, Rosa K, Fu M, et al. Fatigue during treatment for hepatitis C virus: Results of self-reported fatigue severity in two phase Ilb studies of simeprevir treatment in patients with hepatitis C virus genotype 1 infection. BMC Infect Dis 2014;14:465.
65. Manns MP, Fried MW, Zeuzem S, et al. Simeprevir with peginterferon/ribavirin for treatment of chronic hepatitis $C$ virus genotype 1 infection: pooled safety analysis from phase IIb and III studies. J Viral Hepat 2015;22:366-75.

66. Yu M-L, Huang C-F. The unexpected high risk of occurrence or recurrence of hepatocellular carcinoma after successful antiviral therapy with interferonfree direct-acting antivirals. Trans/ Cancer Res 2016;5(Suppl 6):S1111-5.

67. Coffin PO, Stevens AM, Scott JD, et al. Patient acceptance of universal screening for hepatitis C virus infection. BMC Infect Dis 2011;11:160.

68. Myers RP, Crotty P, Town S, et al. Acceptability and yield of birth-cohort screening for hepatitis $C$ virus in a Canadian population being screened for colorectal cancer: a cross-sectional study. CMAJ Open 2015;3:E62-7.

69. White DA, Anderson ES, Pfeil SK, et al. Differences between emergency nurse perception and patient reported experience with an ED HIV and hepatitis C virus screening program. J Emerg Nurs 2016;42:139-45.

70. Barocas JA, Brennan MB, Hull SJ, et al. Barriers and facilitators of hepatitis C screening among people who inject drugs: a multi-city, mixed-methods study. Harm Reduct J 2014;11:1.

71. Norton BL, Voils Cl, Timberlake SH, et al. Community-based HCV screening: knowledge and attitudes in a high risk urban population. BMC Infect Dis 2014;14:74.

72. Zuure FR, Heijman T, Urbanus AT, et al. Reasons for compliance or noncompliance with advice to test for hepatitis $C$ via an internet-mediated blood screening service: a qualitative study. BMC Public Health 2011;11:293.

73. Day CA, White B, Thein $\mathrm{HH}$, et al. Experience of hepatitis $C$ testing among injecting drug users in Sydney, Australia. AIDS Care 2008;20:116-23.

74. Vallabhaneni S, Macalino GE, Reinert SE, et al. Prisoners favour hepatitis C testing and treatment. Epidemiol Infect 2006;134:243-8.

75. Hepatitis C screening in Alberta: a health technology assessment. Calgary: The Health Technology Assessment Unit, University of Calgary; 2016. Available: www. health.alberta.ca/documents/AHTDP-HepatitisC-Screening-HTA-Report-2016.pdf (accessed 2016 Nov. 1).

76. A statement from the pan-Canadian Pharmaceutical Alliance. Toronto: Canada News Wire (CNW) Group Ltd.; 2017. Available: www.newswire.ca/news-releases/ a-statement-from-the-pan-canadian-pharmaceutical-alliance-614373463.html (accessed 2017 Mar. 3).

77. Ontario Drug Benefit Formulary/Comparative Drug Index, edition 42. Toronto: Ontario Ministry of Health and Long-Term Care; 2017. Available: http://www.health. gov.on.ca/en/pro/programs/drugs/formulary42/summary_edition42_20170228. pdf (accessed 2017 Mar. 3).

78. Andrieux-Meyer I, Cohn J, de Araújo ES, et al. Disparity in market prices for hepatitis C virus direct-acting drugs. Lancet Glob Health 2015;3:e676-7.

79. Payne E, Totten S, Archibald C. Hepatitis C surveillance in Canada. Can Commun Dis Rep 2014;40:421. Available: www.phac-aspc.gc.ca/publicat/ccdr-rmtc/14vol40/ dr-rm40-19/assets/pdf/14vol40_19-eng.pdf (accessed 2016 Nov. 1).

80. Andrews JC, Schünemann HJ, Oxman AD, et al. GRADE guidelines: 15. going from evidence to recommendation-determinants of a recommendation's direction and strength. J Clin Epidemiol 2013;66:726-35.

81. A study to characterize the epidemiology of hepatitis C infection in Canada, 2002: final report. Ottawa: Public Health Agency of Canada; 2004.

82. Wong T, Lee S. Hepatitis C: a review for primary care physicians. CMAJ 2006; 174:649-59.

83. Pépin J, Abou Chakra CN, Pépin E, et al. Evolution of the global burden of viral infections from unsafe medical injections, 2000-2010. PLoS One 2014;9:e99677.

84. Trubnikov M, Yan P, Njihia J, et al. Identifying and describing a cohort effect in the national database of reported cases of hepatitis $C$ virus infection in Canada (1991-2010): an age-period-cohort analysis. CMAJ Open 2014;2:E281-7.

85. Remis RS. Modelling the incidence and prevalence of hepatitis $C$ infection and its sequelae in Canada, 2007: final report. Ottawa: Public Health Agency of Canada; 2007. www.phac-aspc.gc.ca/sti-its-surv-epi/model/pdf/model07-eng.pdf (accessed 2016 Oct. 28).

86. Guidelines for the screening, care and treatment of persons with hepatitis $C$ infection: updated version, April 2016. Geneva: World Health Organization; 2016. Available: www.who.int/hepatitis/publications/hepatitis-c-guidelines-2016 (accessed 2016 Sept. 16).

87. Guidelines for the screening, care and treatment of persons with hepatitis $C$ infection. Geneva: World Health Organization; 2014. Available: www.who.int/ hiv/pub/hepatitis/hepatitis-c-guidelines/en (accessed 2016 Sept. 16). 
88. Hepatitis B and C testing: people at risk of infection. London (UK): National Institute for Health and Care Excellence; 2013. Available: www.nice.org.uk/ guidance/ph43 (accessed 2016 Nov. 10).

89. Management of hepatitis C. Edinburgh (UK): Scottish Intercollegiate Guidelines Network; 2013. Available: www.sign.ac.uk/pdf/sign133.pdf (accessed 2016 Sept. 16).

90. Operations directorate, health branch immigration medical examination instructions: hepatitis/liver disease. Ottawa: Citizenship and Immigration Canada; 2013. Available: www.cic.gc.ca/english/department/partner/pp/pdf/IMEI_Hepatitis.pdf (accessed 2016 Oct. 7).

91. Screening for hepatitis $B$ and hepatitis $C$ among ethnic minorities born outside the UK. London (UK): UK National Screening Committee; 2011. Available: https:// legacyscreening.phe.org.uk/hepatitis-ethnic (accessed 2016 Dec. 14).

92. Consensus recommendations for screening and diagnosis. Mulgrave (Australia): Gastroenterological Society of Australia; 2016. Available: http://www. hepcguidelines.org.au/consensus-recommendations/screening-and-diagnosis (accessed 2016 Sept. 16).

93. Pottie K, Greenaway C, Feightner J, et al. coauthors of the Canadian Collaboration for Immigrant and Refugee Health. Evidence-based clinical guidelines for immigrants and refugees. CMAJ 2011;183:E824-925.
94. Final recommendation statement - hepatitis C: screening. Rockville (MD): US Preventive Services Task Force; 2013. Available: www.uspreventiveservicestaskforce.org/ Page/Document/RecommendationStatementFinal/hepatitis-c-screening (accessed 2016 Sept. 16).

95. Recommendations for prevention and control of hepatitis C virus (HCV) infection and HCV-related chronic disease. MMWR Recomm Rep 1998;47:1-39.

96. Smith BD, Morgan RL, Beckett GA, et al. Recommendations for the identification of chronic hepatitis C virus infection among persons born during 19451965. MMWR Recomm Rep 2012;61:1-32.

97. Chou R, Cottrell EB, Wasson N, et al. Screening for hepatitis C virus infection in adults. Report No 12(13)-EHC090-EF. Rockville (MD): US Agency for Healthcare Research and Quality; 2012.

98. Moyer VA. Screening for hepatitis $C$ virus infection in adults: US Preventive Services Task Force recommendation statement. Ann Intern Med 2013;159:349-57.

99. Morgan RL, Baack B, Smith BD, et al. Eradication of hepatitis C virus infection and the development of hepatocellular carcinoma: a meta-analysis of observational studies. Ann Intern Med 2013;158:329-37.

100. Kimer N, Dahl EK, Gluud LL, et al. Antiviral therapy for prevention of hepatocellular carcinoma in chronic hepatitis C: systematic review and meta-analysis of randomised controlled trials. BMJ Open 2012;2:e001313.
This article has been peer reviewed.

Authors: Roland Grad MDCM MSc, Brett D. Thombs PhD, Marcello Tonelli MD SM, Maria Bacchus MD MSc, Richard Birtwhistle MD MSc, Scott Klarenbach MD MSc, Harminder Singh MD MPH, Veronique Dorais MSc, Nathalie M. Holmes BA, Wendy K. Martin PhD, Rachel Rodin MD MPH, Alejandra Jaramillo Garcia MSc

Competing interests: None declared.

Affiliations: Departments of Family Medicine (Grad) and Psychiatry (Thombs), McGill University, Montréal, Que.; Department of Medicine (Tonelli, Bacchus), University of Calgary, Calgary, Alta.; Departments of Family Medicine and Public Health Sciences (Birtwhistle), Queen's University, Kingston, Ont.; Department of Medicine (Klarenbach), University of Alberta, Edmonton, Alta.; Departments of Internal Medicine and Community Health Sciences (Singh), University of Manitoba, Winnipeg, Man.; Public Health Agency of Canada (Dorais, Holmes, Martin, Rodin, Jaramillo Garcia), Ottawa, Ont.

Contributors: Roland Grad, Brett Thombs, Marcello Tonelli, Maria Bacchus, Richard Birtwhistle, Scott Klarenbach, Harminder Singh, Veronique Dorais, Nathalie Holmes, Wendy Martin, Rachel Rodin, Alejandra Jaramillo Garcia, and members of the Task Force not in the guideline working group contributed substantially to the study concept and design, interpretation of the evidence, and revised the draft. Roland Grad, Veronique Dorais and Alejandra Jaramillo Garcia led the analysis and drafted the manuscript. All of the authors gave final approval of the version to be published and agreed to act as guarantors of the work.

Funding: Funding for the Canadian Task Force on Preventive Health Care is provided by the Public Health Agency of Canada. The views of the funding body have not influenced the content of the guideline; competing interests have been recorded and addressed. The views expressed in this article are those of the Task Force and do not necessarily represent those of the Public Health Agency of Canada.

Acknowledgements: We are grateful to Prinon Rahman and Olga Milliken of the Global Health and Guideline Division at the Public Health Agency of Canada, who supported the development of the guideline, and to the Knowledge Translation Program of St. Michael's Hospital for their valuable contributions to patient engagement and knowledge translation work related to this guideline. The authors would like to thank CADTH for its systematic review on HCV screening, which supported this guideline, as well as the authors of the systematic review on HCV treatment (Francesca Reyes Domingo, Nathalie M. Holmes, Rana Rahal, Mitulika Chawla, Kristin Klein and Alejandra Jaramillo Garcia). The authors would like to thank Dr William W.L. Wong (School of Pharmacy, University of Waterloo) and Dr Naveed Zafar Janjua (British Columbia Centre for Disease Control, School of Population and Public Health, University of British Columbia) for their involvement as clinical experts and peer reviewers. The following organizational and peer reviewers provided thoughtful comments that helped to improve the quality of this manuscript: Songul BozatEmre PhD, Council of Chief Medical Officers of Health (CCMOH), Active Living, Population and Public Health Branch, Manitoba Health, Seniors and Active Living, Assistant Professor, Max Rady College of Medicine, University of Manitoba; Aysegul Erman, Leslie Dan Faculty of Pharmacy, University of Toronto; Jennifer Fournier NP-PHC MHS, Canadian Association of Advanced, Practice Nurses; Dr Jack Janvier MD FRCPC, Division of Infectious Diseases, University of Calgary, Peter Lougheed Center; Dr Claude Laberge, médecin-conseil, Direction générale de la santé publique, Ministère de la santé et des services sociaux, Québec; Rebecca L. Morgan, McMaster University; Dr. Giulia-Anna Perri MD CCFP(PC)(COE), College of Family Physicians of Canada, Baycrest Health Sciences, Division of Palliative Care, Department of Family and Community Medicine, University of Toronto; Dr. Daniel C. Sadowski MD FRCP(c), Division of Gastroenterology, University of Alberta, Canadian Association of Gastroenterology; Dena Schanzer, Infectious Disease Prevention and Control Branch, Public Health Agency of Canada; Denise H. Werker MD MHSC FRCPC, Government of Saskatchewan, Deputy Chief Medical Health Officer, Ministry of Health; and David KH Wong MD FRCPC, Toronto Centre for Liver Disease, University of Toronto.

Correspondence to: Canadian Task Force on Preventive Health Care, info@canadiantaskforce.ca 